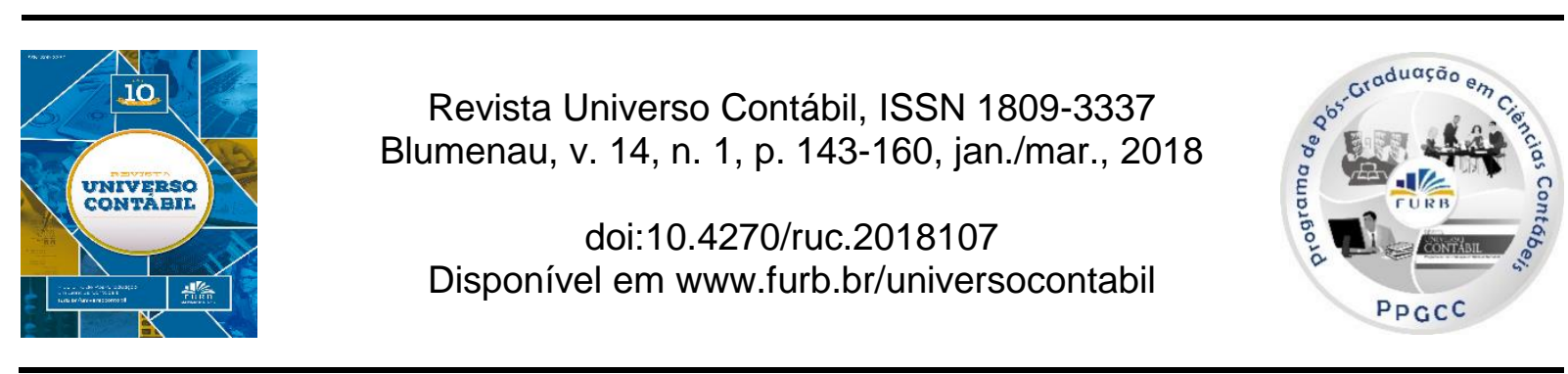

\title{
DIVIDENDOS, COMPOSIÇÃO DE CARTEIRAS E PERFORMANCE DE FUNDOS DE AÇÕES $^{1}$
}

\section{DIVIDENDS, PORTFOLIO COMPOSITION AND PERFORMANCE OF MUTUAL FUNDS}

\section{DIVIDENDOS, COMPOSICIÓN DE CARTERAS Y EL RENDIMIENTO DE LOS FONDOS DE ACCIONES}

\author{
Érica Juvercina Sobrinho \\ Mestrado em Ciências Contábeis pela Universidade Federal de Uberlândia \\ Endereço: Av. João Naves de Ávila, 2121, Bloco F, Sala 1F-248 - Santa Mônica \\ CEP: 38400-902 - Uberlândia - MG - Brasil \\ E-mail: ericajuvercina@hotmail.com \\ Telefone: (34) 3291-5904.
}

\begin{abstract}
Rodrigo Fernandes Malaquias
Doutor em Administração pela Fundação Getúlio Vargas Professor dos PPGs em Ciências Contábeis e Administração da Universidade Federal de Uberlândia Endereço: Av. João Naves de Ávila, 2121, Bloco F, Sala 1F-216 - Santa Mônica CEP: 38400-902 - Uberlândia - MG - Brasil

E-mail: rodrigofmalaquias@yahoo.com.br

Telefone: (34) 3239-4132.
\end{abstract}

\section{RESUMO}

O objetivo deste estudo foi analisar a relação da distribuição de dividendos com a composição da carteira e a performance dos fundos mútuos brasileiros de ações no período de 2009 a 2015. Por meio de uma análise com dados em painel decorrente da utilização de variáveis delineadas por Fama e French $(1993,2015)$ e Carhart (1997), identificou-se indícios de que os fundos de investimentos em ações (em média) utilizam a informação proveniente de dividendos para decidir sobre a composição de suas carteiras apesar de priorizarem o fator mercado como sinalizador para a tomada de decisões. Portanto, houve indícios de uma relação positiva entre a distribuição de dividendos e a composição das carteiras dos fundos da amostra. Por outro lado, os resultados indicam que não necessariamente a utilização desta informação é um fator que contribui positivamente para um melhor retorno ajustado ao risco dos fundos mútuos.

Palavras-Chave: fundos mútuos, Índice de Sharpe, portfólio.

\footnotetext{
${ }^{1}$ Artigo recebido em 13/12/2017. Revisado por pares em 13/08/2018. Reformulado em 14/11/2018. Recomendado para publicação em 27/11/2018 por Tarcísio Pedro da Silva. Publicado em 20/12/2018. Organização responsável pelo periódico: FURB.
} 


\section{ABSTRACT}

The aim of this paper was to analyze the relationship between the distribution of dividends, the portfolio composition and the mutual fund's performance between the period from 2009 to 2015. Through an analysis with panel data resulting from the use of variables from Fama and French (1993, 2015) and Carhart (1997), we observed some evidences that investment funds of the sample (on average) use information from dividends to decide about portfolio composition, despite prioritizing the market factor as a signal for decision-making. Therefore, we found evidence about a positive relationship between dividends and portfolio composition of the funds included in the sample. On the other hand, the results indicate that not necessarily the use of this information is a factor that contributes positively to a better riskadjusted return of the mutual funds.

Keywords: mutual funds, Sharpe Ratio, portfolio.

\section{RESUMEN}

El objetivo de este estudio fue analizar la relación de la distribución de dividendos con la composición de las carteras y el rendimiento de los fondos de acciones brasileños en el período de 2009 a 2015. Por medio de un análisis con datos en panel resultante de la utilización de variables delineadas por Fama y French $(1993,2015)$ y Carhart (1997), se identificó que los fondos de inversiones (en promedio) utilizan la información proveniente de dividendos para decidir sobre la composición de sus carteras a pesar de priorizar el factor mercado como indicador para la toma de decisiones. De esta forma, observamos una relación positiva entre la distribución de dividendos y la composición de las carteras de los fondos de la muestra. Por otro lado, los resultados indican que no necesariamente la utilización de esta información es un factor que contribuye positivamente a un mejor retorno ajustado al riesgo de los fondos mutuos. Palabras clave: fondos de acciones, Índice de Sharpe, cartera.

\section{INTRODUÇÃO}

Os fundos de ações estão sujeitos à influência de anomalias e imperfeições do mercado financeiro, o que afeta a precificação e retorno do ativo compondo seu portfólio. Com o intuito de capturar o caráter preditivo do desempenho futuro dos fundos mútuos, diversas técnicas e modelos matemáticos tentam mensurar alterações no grau de risco e retorno, mas a literatura ainda não apresenta um consenso destes fatores induzindo os participantes do mercado a adotar uma variedade de estratégias de negociação em busca do equilíbrio entre risco e retorno.

Mudanças inesperadas na distribuição de dividendos transmitem informações sobre o nível de fluxos de caixa atuais e futuros das empresas (DENIS; DENIS; SARIN, 1994) e, consequentemente, a demanda pelos ativos responde proporcionalmente. Dado que o sistema financeiro tem uma dinâmica de transformações bruscas na geração de riqueza, a política de dividendos pode consolidar-se como um parâmetro estratégico aos fundos mútuos, já que, seu comportamento, quanto à manutenção de ações em suas carteiras, pode ser reflexo da instabilidade econômica, principalmente em mercados emergentes.

Segundo Bhattacharya (1979), dividendos funcionam como uma sinalização antecipada do fluxo de caixa empresarial, reduzindo a assimetria informacional. Assim, a sinalização transmitida pelos dividendos pode ser considerada como instrumento válido aos gestores de fundos conduzindo informações sobre expectativas de desenvolvimento financeiro de uma empresa. Estudiosos como Benartzi, Michaely e Thaler (1997), Brealey, Myers e Allen (2011), Procianoy e Verdi (2009) e Scholz (1992) apoiam a teoria da sinalização dos dividendos, cujas alterações acarretam movimentação no valor das ações. 
Como não foi identificado um estudo anterior que formalizasse a análise empírica sobre a aplicação (ou não) dos dividendos como informação relevante para a composição das carteiras de fundos de investimento e sua performance, há uma lacuna no âmbito acadêmico referente ao tema. Dessa forma, o trabalho vem analisar e responder a seguinte questão: Qual a relação entre distribuição de dividendos das empresas investidas com a composição das carteiras e a performance dos fundos mútuos de ações? Com base nessas considerações, este estudo apresenta por objetivo principal analisar a relação da distribuição de dividendos com a composição das carteiras e a performance dos fundos mútuos de ações no período de 2009 a 2015.

A literatura sobre a política de dividendos tem produzido resultados divergentes quanto à criação de valor ao investidor, diante do paradoxo entre retenção de lucros em prol de novos investimentos (DENIS et al., 1994; GRAHAM; DODD, 1988; RAMALINGEGOWDA; WANG; YU, 2013). A pesquisa sobre dividendo desperta interesse na literatura pela sua complexidade, desde o estudo seminal de Lintner (1956), pelo duplo papel, uma vez que pode atuar como políticas de desenvolvimento e crescimento da empresa ou também podendo atuar como um canal de comunicação entre a empresa e o mercado (MOREIRAS; TAMBOSI FILHO; GARCIA, 2012).

A natureza da investigação do presente estudo fornece evidências sobre a hipótese de que alterações nos níveis de pagamento de dividendos transmitem informações importantes aos participantes do mercado. Ademais, o presente estudo promove alguma evidência adicional pertinente à literatura existente, especificamente no estudo de Fama e French (1993, 2015), sobre o portfólio dos fundos mútuos. Desta forma, permite considerar se a política de dividendos é um parâmetro corporativo importante à tomada de decisões dos fundos, uma vez que representa um componente do retorno do investimento total (NIKOLAOS; EVANGELOS; DIMITRIOS, 2010).

\section{HIPÓTESES DE ESTUDO}

\subsection{Eficiência de Mercado e Sinalização}

É fato que a gestão corporativa detém maior poder informativo sobre as expectativas financeiras das empresas em relação aos investidores e, segundo Vieira, Pinho e Leite (2013, p. 13), "de acordo com a teoria da sinalização ou hipótese do conteúdo informativo dos dividendos, espera-se que o anúncio de alteração dos dividendos esteja positivamente relacionado com a reação do preço das ações e com os resultados futuros das empresas". Entretanto, de acordo com a análise empírica de Santos et al. (2008), as mudanças de dividendos não necessariamente implicam em informações interpretadas pelo mercado financeiro como persistência de mudanças passadas dos lucros. Segundo os autores, "o sinal das mudanças de dividendos também não é interpretado como conteúdo informacional acerca da persistência das mudanças precedentes dos lucros" (SANTOS et al., 2008, p. 15).

Considerando que investidores externos possuem informações incompletas sobre fluxos de caixa futuros, o pagamento de dividendos pode funcionar como um sinal, assim como postulado por Lintner (1956), Myers e Majluf (1984a) e Grullon, Michaely e Swaminathan (2002) por meio de informação superior transmitida ao mercado. Adicionalmente, a presença de investidores institucionais tem um efeito positivo e significativo sobre a liquidez do mercado de ações, o que confirma o efeito da sinalização no mercado (AJINA; LAKHAL; SOUGNÉ, 2015), ou seja, negociam ativos em grandes proporções, reduzindo custos de transação e melhorando a liquidez.

Vale ressaltar o tradeoff entre investimentos e dividendos no âmbito corporativo, em que os gestores dos fundos, ao analisarem as empresas, podem levar em consideração essas 
informações para sua tomada de decisão. Graham e Dodd (1988) destacam a importância da política de dividendos nos impactos sobre o preço da ação, mas reconhecem o paradoxo do "valor é aumentado por tirar valor", assim como apontado por Jabbouri (2016) como uma relação negativa entre fluxo de caixa livre e pagamento de dividendos. O fluxo de caixa livre é a base para distribuição de dividendos e sua utilização eficaz está atrelada ao papel dos investidores institucionais supervisionando as empresas (DENG; CHAN; YANG; CAUDWELL, 2015). Portanto, uma política de dividendos ótima pode ser aquela que equilibra, da melhor forma possível, a quantidade que será retida pela empresa e aquela a ser distribuída pelos acionistas na forma de dividendos (GONZÁLEZ, 1998). Esse conteúdo remete à construção da hipótese H1:

H1a: Existe relação entre a distribuição de dividendos e a alocação de recursos em ações por parte dos fundos mútuos de ações no Brasil.

O estudo de DeAngelo e DeAngelo (1990) abordou os ajustes da política de dividendos entre 1980 a 1985 (período de turbulência financeira). Elevada incidência de reduções de dividendos demonstrou a tentativa de ajustes graduais em momentos de angustia. Achados de Benartzi, Michaely e Thaler (1997) confirmam que aumentos nos dividendos implicam em aumentos significativos nos resultados da empresa apesar de não prever os mesmos resultados no futuro. Em geral, empresas que aumentam os dividendos apresentam retornos excedentes significativos para o triênio seguinte, o que é suporte empírico para o ponto de vista de que os dividendos fornecem informação sobre os fluxos de caixa futuros assim como Watts (1973) afirmou pioneiramente.

Mudanças na política de dividendos estão fortemente correlacionadas com mudanças nos ganhos simultaneamente, ou seja, ganhos levam a dividendos e não vice-versa (BENARTZI et al., 1997). Baker e Wurgler (2004) sugerem que os gerentes corporativos aparentemente reconhecem e atendem às mudanças na demanda dos investidores por empresas pagadoras de dividendos. Em momentos de turbulência econômica, há evidências de redução precoce e agressiva de dividendos além de relutância de gestores corporativos à omissão de pagamentos (DEANGELO; DEANGELO, 1990). A explicação é que os dividendos, em grande parte, estão reagindo a mudanças passadas de lucros, e não como preditores de ganhos futuros, ao apresentar caráter informacional sobre o presente (BENARTZI et al., 1997), o que fundamenta a parte b da primeira hipótese do estudo.

H1b: Existe relação entre a alteração nos níveis de distribuição de dividendos e a atratividade de ações por parte dos fundos mútuos de ações no Brasil.

\subsection{Teoria do Portfólio}

Um dos maiores avanços na área de investimentos é o reconhecimento da impossibilidade de criar uma carteira de investimentos ideal pela simples combinação de inúmeros títulos com características de risco-retorno desejáveis, pois a combinação desses ativos interfere no desempenho ótimo, conforme demonstrado na Teoria do Portfólio (REILLY; BROWN, 2012), amplamente conhecida como a Moderna Teoria de Finanças. A análise da correlação entre ativos no portfólio é de grande relevância na investigação de Markowitz (1952), já que possibilitou identificar os benefícios provenientes da diversificação e seu impacto na relação entre risco e retorno de investimentos, o que culminou no desenvolvimento de modelos aprimorados em estudos posteriores.

Os modelos alfa de Jensen (1968), de Carhart (1997), Sharpe (1966), de precificação CAPM (Capital Asset Pricing Model) e de Fama e French (1993) predominam na literatura (BORGES; MARTELANC, 2015; CARHART, 1997; CASTRO; MINARDI, 2009; DANIEL; 
GRINBLATT; TITMAN; WERMERS, 1997; DÍAZ-MENDOZA; LÓPEZ-ESPINOSA; MARTÍNEZ, 2014; GIL-BAZO; RUIZ-VERDÚ, 2009; GUERCIO; TKAC, 2002; JAIN; WU, 2000) e são amplamente utilizados, permitindo refinamento e avanço dos estudos em finanças. Apesar disso, as ferramentas delineadas até o momento podem possuir limitações em sua efetiva aplicação, sendo constantemente aprimoradas para atender as necessidades dos investidores.

Nesse sentido, o desempenho do fundo pode estar atrelado a anomalias pouco exploradas em metodologias empíricas, como a distribuição de dividendos. Para tanto, Calvet e Fisher (2008) destacam que o campo de gestão de carteiras e de precificação de ativos requer visão precisa das propriedades estatísticas dos retornos dos ativos para quantificar a exposição de riscos e adequar o portfólio. Considerando que a literatura tem se aprimorado nas anomalias de mercado, isso instiga a examinar uma variável relacionada a dividendos para tentar capturar o retorno anormal dos fundos no mercado brasileiro. Dessa forma, formulou-se a hipótese:

H2a: existe relação entre a política de dividendos das empresas investidas e o desempenho dos fundos.

Diante de uma variável detentora de complexidade como o dividendo (MOREIRAS et al., 2012), qualquer análise direcionada à performance dos fundos exige ponderação ao longo do tempo. Segundo Lintner (1956), há uma tendência das empresas em adotar uma política de dividendos linear, aumentando o montante somente quando os incrementos dos lucros demonstrarem ser "permanentes", de forma que reduzir dividendos não é uma alternativa adequada para as empresas. Em uma economia emergente como a brasileira, o dividendo pode fazer diferença na performance dos fundos mútuos devido ao sinal transmitido, induzindo a uma reavaliação do portfólio. Nesse contexto, mudanças nos dividendos podem sinalizar alguma informação com relação ao presente, mas também podem sinalizar algo sobre condições futuras de sustentabilidade institucional, bem como de retorno aos investidores. Assim, surge a hipótese:

H2b: existe relação entre a variação nos níveis de dividendos e o desempenho dos fundos de investimentos.

\section{ASPECTOS METODOLÓGICOS}

\subsection{Tipologia e Amostra}

O presente trabalho objetiva analisar a relação da distribuição de dividendos com a atratividade dos fundos mútuos de ações e, posteriormente, com a performance (retorno ajustado ao risco) desses investidores institucionais no mercado brasileiro. Os dados coletados envolvem todos os fundos de investimentos em ações com dados disponíveis para o período correspondente a 2009 até 2015, no banco de dados Economática ${ }^{\circledR}$. A escolha da amostra se justifica pela concentração de portfólio voltada para ativos mobiliários em ações, o que viabiliza o estudo do comportamento dos fundos frente ao pagamento de dividendos pelas empresas investidas.

Alguns critérios foram adotados com o objetivo de qualificar a amostra que, inicialmente, apresentava 4.000 fundos de investimentos em ações. Entretanto, considerou-se apenas os fundos que apresentaram investimentos em ações de empresas brasileiras (fundos domésticos) e disponibilizavam informações para tamanho, taxa de administração, taxa de performance e período de lock-up (variáveis de controle), bem como para a rentabilidade mensal (variável utilizada para calcular o Índice de Sharpe de cada fundo). Isso resultou em uma amostra final de 1.479 fundos. 
Desmembrou-se o conteúdo das carteiras de cada fundo com o intuito de identificar cada ativo e verificar onde estavam alocados. Nesse momento, foi necessário recorrer novamente à base de dados Economática ${ }^{\circledR}$, contendo informações das ações negociadas por empresas na BM\&FBovespa, para identificar cada ativo pela utilização de seu respectivo código na bolsa. Além disso, selecionou-se todas as empresas e respectivas informações sobre dividendos totais pagos no ano, tamanho (valor de mercado), patrimônio líquido, lucro líquido, liquidez em bolsa, ativo total, beta. Essas informações foram utilizadas para delinear as variáveis do modelo de Fama e French $(1993,2015)$ e Carhart (1997) escolhidas pela disseminação na literatura.

Foram excluídas da amostra empresas financeiras, em linha com os estudos já realizados anteriormente sobre o assunto (FAMA; FRENCH, 1993, 2015; FORTI; PEIXOTO; ALVES, 2015; SILVA, 2004), bem como ações que não apresentavam dados suficientes para construção das variáveis em todos os períodos analisados. Ao finalizar a seleção das ações, obteve-se, em média, 175 empresas com dados disponíveis por ano (um total de 1.227 observações de empresas para o período).

\subsection{Variáveis}

Para investigar se a carteira dos fundos está relacionada com atratividade das ações que pagam dividendos e o respectivo impacto na performance dos fundos, foram consideradas variáveis adotadas pelo modelo de Fama e French $(1993,2015)$, incluindo o fator momentum (Carhart, 1997), seguindo a metodologia na formação das variáveis com as devidas adaptações ao mercado brasileiro.

Variáveis Dependentes:

- PH (Portfolio Holding) é a variável dependente utilizada para a hipótese H1 desse estudo e é calculada como o somatório do porcentual do portfólio dos fundos alocado em cada ação.

- SHARPE (Índice de Sharpe) é a variável dependente utilizada para as hipóteses de H2 e refere-se à performance do fundo (Sharpe) ao final do ano. Consiste na razão entre o retorno médio do fundo acima da taxa livre de risco, ponderado pela sua volatilidade. Foi adotado o parâmetro indicado por Israelsen (2005), no que se refere a multiplicar o prêmio pelo risco pela volatilidade quando o retorno do período for inferir à taxa livre de risco.

Variáveis independentes

- LIQ (Liquidez em Bolsa) representa uma medida baseada no fator mercado de Fama e French (1993, 2015). Foi considerada como estimativa para as empresas que representam o retorno de mercado aquelas com maior liquidez em bolsa. Para as hipóteses de $\mathrm{H} 1$, este indicador é calculado pela liquidez em bolsa do ativo ao final de cada ano, sendo uma variável dummy equivalendo a 1 se a empresa investida i possui liquidez acima da mediana e 0 caso contrário. Já para as hipóteses de H2, a dummy é multiplicada pelo somatório do percentual do portfólio do fundo alocado com base nesse indicador, gerando uma variável escalar para análise das hipóteses $\mathrm{H} 2 \mathrm{a}$ e $\mathrm{H} 2 \mathrm{~b}$.

- BOOK (Book-to-Market) representa a relação entre o valor patrimonial pelo valor de mercado da empresa. Para análise das hipóteses de H1, esse índice foi construído com dados ao final de cada ano, sendo uma variável dummy correspondente a 1 se a empresa investida i possui book-to-market acima da mediana e 0 caso contrário. Com relação às hipóteses de $\mathrm{H} 2$, a dummy é multiplicada pelo somatório do percentual do portfólio do fundo alocado com base nesse indicador, gerando uma variável escalar para análise das hipóteses H2a e H2b. 
- VM (Valor de Mercado) representa o fator tamanho e está relacionada à dimensão. Esta variável foi inserida para captar o efeito tamanho como uma variável dummy. Para as hipóteses de H1, assume valor 1 para empresas com valor de mercado acima da mediana (por ano) e zero para empresas com valor de mercado abaixo da mediana. Posteriormente, a dummy é multiplicada pelo somatório do percentual do portfólio do fundo alocado com base nesse indicador, gerando uma variável escalar para análise das hipóteses H2a e H2b. Esta classificação é realizada anualmente, considerando o valor de mercado das empresas no último dia de cada ano (31/12).

- RETP (Retorno Passado) representa o fator Momentum. É calculada a partir do retorno passado dos ativos (nos últimos 12 meses). Neste estudo, é representada por uma variável dummy em que 1 equivale às ações que superaram a mediana, por ano, no que se refere ao retorno médio dos últimos 12 meses e 0 , caso contrário. A dummy é multiplicada pelo somatório do percentual do portfólio de todos os fundos alocado com base nesse indicador, gerando uma variável escalar para análise das hipóteses $\mathrm{H} 2 \mathrm{a}$ e $\mathrm{H} 2 \mathrm{~b}$.

- INV (Investimentos) representa o fator investimentos. Seu cálculo baseia-se no crescimento do ativo total anual (FAMA; FRENCH, 2015). Neste estudo, sua mensuração como característica das empresas está representada por uma variável dummy, para as hipóteses de $\mathrm{H} 1$, recebendo valor 1 para empresas com novos investimentos acima da mediana e 0 caso contrário. Assim, esta variável representa, quando 1, empresas que pertencem ao grupo com maior investimento em cada ano e, quando 0 , empresas que pertencem ao grupo com menor investimento em cada ano. A dummy é multiplicada pelo somatório do percentual do portfólio do fundo alocado com base nesse indicador, gerando uma variável escalar para análise das hipóteses $\mathrm{H} 2 \mathrm{a}$ e H2b.

- RENT (Rentabilidade) representa o fator rentabilidade (FAMA; FRENCH, 2015). Para análise das hipóteses H1a e H1b, esse atributo das empresas é mensurado por meio de uma variável dummy, sendo o valor 1 para empresas com rentabilidade acima da mediana e 0 para empresas com menor rentabilidade anual. A dummy é multiplicada pelo somatório do percentual do portfólio do fundo alocado com base nesse indicador, gerando uma variável escalar para análise das hipóteses H2a e H2b.

- DIV equivale à razão entre dividendo pago total anual dividido pelo ativo total em 31/12 de cada ano, representando o tamanho relativo do fluxo de caixa distribuído aos acionistas (FORTI et al., 2015). Para as hipóteses H1a e H1b, esse atributo das empresas é mensurado por meio de uma variável dummy, sendo o valor 1 para empresas com dividendos acima da mediana e 0 caso contrário. A dummy é multiplicada pelo somatório do percentual do portfólio do fundo alocado com base nesse indicador, gerando uma variável escalar para análise das hipóteses H2a e H2b.

- DIVADIC (Dividendo adicional) equivale à diferença entre a razão do dividendo pago total anual dividido pelo ativo total em 31/12 de cada ano com a razão do dividendo pago total anual dividido pelo ativo total em 31/12 do ano anterior. Esse atributo é mensurado por meio de uma variável dummy, sendo o valor 1 para empresas com índice positivo (ou seja, empresas em que o dividendo pago no ano $\mathrm{t}$ é proporcionalmente maior do que o dividendo pago no ano t-1) e 0 para empresas com índice negativo ou nulo. O objetivo dessa variável é captar a variação do dividendo, ou seja, identificar a alteração do dividendo ao longo do período.

- BETA equivale à razão entre a covariância do ativo com o índice de mercado (Ibovespa) pelo seu desvio padrão, para medir o risco não diversificável. O índice já calculado pela base de dados Economática ${ }^{\circledR}$ mede a variação de uma ação em relação a uma carteira de mercado diversificada (IBOVESPA). 
- Características do fundo podem influenciar a atratividade pelos ativos a serem investidos e até mesmo na performance do próprio fundo. Por isso, foram utilizadas outras variáveis:

- LNPL (logaritmo neperiano médio do patrimônio líquido) que representa o tamanho do fundo. Para minimizar os efeitos provenientes de diferenças escalares, utilizou-se o logaritmo neperiano do patrimônio do fundo.

- AGE (age) representa a idade do fundo em anos, já que os fundos mais recentes podem ter aumentado as necessidades de negociação (Evans, 2008). Refere-se à indicação do tempo (ano) de existência que este fundo está cadastrado na CVM (GUARANA, 2012).

- TXADM (Taxa de Administração) representa a taxa máxima de administração cobrada pelo fundo. Essa variável é mensurada pelo maior percentual cobrado no período de 12 meses.

- TXPERF (Taxa de Performance) representa a taxa de performance cobrada pelo fundo e refere-se a um percentual cobrado quando a rentabilidade do fundo supera um parâmetro de referência (benchmark). Essa variável é mensurada por meio de uma dummy, recebendo 1 para fundos que cobram taxa de performance e 0 para os demais casos.

- LOCKUP (lockup) foi incluída pela sua relação com diversificação no portfólio dos fundos, já que, representa o período de bloqueio que o investidor deve alocar o capital. É mensurada pelo montante de dias total requerido pelo investidor para avisar o fundo sobre o resgate e o pagamento de sua cota.

\subsection{Procedimentos quantitativos}

O banco de dados foi estruturado em painel para explorar características cross-section ao longo do tempo. Para testar o método com melhor adaptação aos dados, foram estimados os modelos por meio de testes econométricos utilizando os testes de Breush e Pagan, Hausman e Chow, fundamentando-se em Fávero (2015). Quanto à análise, o modelo proposto visa testar a atratividade dos fundos de investimentos pela aquisição de ações pagadoras de dividendos com a inclusão da variável DIV. Embora Fama e French (1993) e Carhart (1997) não utilizem essa variável em seu modelo, Fama e French (1988) analisaram o dividend yield, o que permitiu definir o modelo para testar a hipótese H1a. Para o teste de H1b, a variável DIV foi substituída pela variável DIVADIC.

Posteriormente, investiga-se a relação dos dividendos na performance dos fundos alterando a variável dependente para a performance do fundo (SHARPE) com a finalidade de testar a hipótese H2a. O mesmo procedimento de substituição da variável DIV foi realizado, para inclusão da variável DIVADIC, permitindo assim o teste de $\mathrm{H} 2 \mathrm{~b}$. Os procedimentos quantitativos foram realizados por meio do software Stata.

\section{RESULTADOS}

Diante da metodologia descrita anteriormente, gerou-se uma estatística descritiva das variáveis dos fundos e das respectivas empresas investidas no período de 2009 a 2015 conforme Tabelas 1 e 2.

Para as variáveis relacionadas (Tabela 1) às características das empresas investidas pelos fundos mútuos, pode-se destacar portfólio holding $(\mathrm{Ph})$ que apresentou valores de grandes dimensões, decorrente do somatório do montante investido pelos fundos. A variável DIVADIC se difere das demais por não estar atrelada à mediana (em comparação com as demais empresas da amostra) para a sua definição. Demais dados relativos às variáveis independentes apresentaram desvio padrão correspondente à variável binária. É oportuno também ressaltar o 
valor zero na coluna "mínimo", indicando que, durante o período, existe pelo menos uma ação que não recebeu alocações de recursos de nenhum dos fundos que compõe a amostra do estudo.

Tabela 1. Estatística descritiva das empresas investidas pelos fundos mútuos

\begin{tabular}{cccccc}
\hline Variáveis & $\mathbf{N}$ & Méd. & Desv. Pad. & Mín. & Máx. \\
\hline$P H$ & 1,227 & 298.881 & 721.913 & 0.000 & $6,332.589$ \\
\hline$L I Q$ & 1,227 & 0.500 & 0.500 & 0.000 & 1.000 \\
\hline$V M$ & 1,227 & 0.500 & 0.500 & 0.000 & 1.000 \\
\hline$B O O K$ & 1,227 & 0.500 & 0.500 & 0.000 & 1.000 \\
\hline$R E T P$ & 1,227 & 0.500 & 0.500 & 0.000 & 1.000 \\
\hline$I N V$ & 1,227 & 0.500 & 0.500 & 0.000 & 1.000 \\
\hline$R E N T$ & 1,227 & 0.500 & 0.500 & 0.000 & 1.000 \\
\hline$D I V$ & 1,227 & 0.500 & 0.500 & 0.000 & 1.000 \\
\hline$D I V A D I C$ & 1,227 & 0.393 & 0.489 & 0.000 & 1.000 \\
\hline$B E T A$ & 1,227 & 0.500 & 0.500 & 0.000 & 1.000 \\
\hline
\end{tabular}

Na Tabela 2, tem-se um painel com a estatística descritiva combinando todos os dados tanto de empresas investidas quanto dos fundos mútuos. Vale destacar que a variável dependente para $\mathrm{H} 2 \mathrm{a}$ e $\mathrm{H} 2 \mathrm{~b}$, performance do fundo (SHARPE), se distingue das demais ao assumir valores negativos.

Tabela 2. Estatística descritiva dos fundos de ações

\begin{tabular}{cccccc}
\hline Variáveis & $\mathbf{N}$ & Méd. & Desv. Pad. & Mín. & Máx. \\
\hline LIQ & 6,449 & 43.531 & 31.051 & 0.000 & 99.967 \\
\hline$V M$ & 6,449 & 41.853 & 30.759 & 0.000 & 99.958 \\
\hline BOOK & 6,449 & 23.121 & 27.421 & 0.000 & 99.924 \\
\hline RETP & 6,449 & 17.480 & 18.505 & 0.000 & 95.586 \\
\hline INV & 6,449 & 30.682 & 27.130 & 0.000 & 99.913 \\
\hline RENT & 6,449 & 24.943 & 22.378 & 0.000 & 99.810 \\
\hline DIV & 6,449 & 32.213 & 27.320 & 0.000 & 99.917 \\
\hline DIVADIC & 6,449 & 19.149 & 20.320 & 0.000 & 99.694 \\
\hline BETA & 6,449 & 23.084 & 23.325 & 0.000 & 99.903 \\
\hline SHARPE & 6,449 & -6.066 & 8.960 & -46.185 & 1.861 \\
\hline LNPL & 6,449 & 17.167 & 1.705 & 9.943 & 23.356 \\
\hline AGE & 6,449 & 6.658 & 5.517 & 0.339 & 34.494 \\
\hline Lockup & 6,449 & 9.992 & 21.879 & 0.000 & 180.000 \\
\hline TXADM & 6,449 & 1.419 & 1.176 & 0.000 & 5.500 \\
\hline TXPERF & 6,449 & 0.366 & 0.482 & 0.000 & 1.000 \\
\hline
\end{tabular}

Ainda na Tabela 2, em média, os fundos da amostra investem grande parte de seu portfólio em ações baseando-se nos fatores LIQ (43,5\%), VM (41,8\%) e DIV (32,2\%). De modo ilustrativo, é possível observar que os fundos da amostra investem cerca de 32,2\% de seu portfólio em ações que receberam 1 no fator dividendo. Por outro lado, há fundos que não investem nada em empresas que receberam 1 no fator dividendo (pois o valor mínimo é zero) e há fundos que investem até $99,9 \%$ de suas carteiras em empresas que receberam 1 no fator dividendo. Cabe salientar que fundos com desempenho negativo se sobressaem na amostra, conforme evidenciado na variável SHARPE. 
Resultados apresentados na Tabela 3 para as hipóteses H1a e H1b, alocação de recursos dos fundos em ações, evidenciam três análises com a variável dependente PH. Os modelos 01 , 02 e 03 diferem-se em função das variáveis independentes que fazem parte de cada um deles.

Tabela 3. Análise da atratividade das ações pelos fundos de ações entre 2009 e 2015

\begin{tabular}{|c|c|c|c|c|c|c|c|c|c|}
\hline \multirow{2}{*}{$\begin{array}{l}\text { Painel: } \\
\text { Variáveis }\end{array}$} & \multicolumn{3}{|c|}{01} & \multicolumn{3}{|c|}{02} & \multicolumn{3}{|c|}{03} \\
\hline & Coef. & $\mathbf{t}$ & $P>t$ & Coef. & $\mathbf{t}$ & $P>t$ & Coef. & $\mathbf{t}$ & $P>t$ \\
\hline LIQ & 30.992 & 0.89 & 0.373 & 35.586 & 1.02 & 0.307 & --- & --- & --- \\
\hline VM & 80.417 & 2.13 & 0.034 & 81.001 & 2.13 & 0.033 & 87.297 & 2.34 & 0.019 \\
\hline BOOK & -132.128 & -4.87 & 0.000 & -133.184 & -4.90 & 0.000 & -133.268 & -4.95 & 0.000 \\
\hline INV & 8.777 & 0.49 & 0.626 & 7.942 & 0.44 & 0.662 & --- & --- & --- \\
\hline RENT & 64.292 & 2.73 & 0.007 & 70.967 & 3.02 & 0.003 & 66.061 & 2.81 & 0.005 \\
\hline RETP & -10.448 & -0.61 & 0.543 & -7.436 & -0.43 & 0.665 & --- & --- & --- \\
\hline BETA & -2.852 & -0.17 & 0.869 & -2.950 & -0.17 & 0.865 & --- & --- & --- \\
\hline DIV & 64.033 & 2.65 & 0.008 & --- & --- & --- & 62.883 & 2.62 & 0.009 \\
\hline DIVADIC & --- & --- & --- & 24.733 & 1.40 & 0.163 & --- & --- & --- \\
\hline _cons & 247.381 & 6.84 & 0.000 & 263.224 & 7.40 & 0.000 & 257.428 & 8.69 & 0.000 \\
\hline
\end{tabular}

Nota: Variável dependente Portfolio Holding (PH) relacionada com as variáveis independentes: liquidez em bolsa (LIQ), valor de mercado (VM), book-to-market (BOOK), investimentos (INV), rentabilidade (RENT), retorno passado (RETP), risco sistemático (BETA), dividendos (DIV) e dividendo adicional (DIVADIC)

Diante da Tabela 3, os coeficientes das variáveis BOOK, RENT e DIV foram significativos a 1\%. Considerando o fator dividendo (DIV), houve significância para empresas que tendem a distribuir elevados montantes, apontando para a não rejeição da hipótese H1a. É um atributo importante aos gestores dos fundos, uma vez que, o dividendo sinaliza uma predição do desempenho da empresa, como já discutido anteriormente (BAKER, 2009; DEANGELO; DEANGELO; SKINNER, 1992; DEANGELO; DEANGELO, 1990; VAN HORNE; WACHOWICZ, 2005), e possibilita adequações no portfólio. Os dividendos pagos em uma ação podem fornecer um fluxo de renda mais barato do que controlar uma venda de ativo (DENIS et al., 1994), pois os custos de transação podem superar o retorno. Imperfeições do mercado, como custos de transação, impostos e restrições normativas, podem induzir os fundos de investimento em ações a um efeito clientela de dividendos (BAKER; NAGEL; WURGLER, 2007; BLACK; SCHOLES, 1974). Se empresas de alto rendimento também atraem investidores com preferência por dividendos generosos (DENIS et al., 1994), a magnitude de resposta no preço das ações a um anúncio de mudança nos dividendos será positivamente relacionada com a amplitude dos dividendos a distribuir.

Sobre a hipótese H1b não é possível afirmar sua relevância para a atratividade dos fundos, o que implica em não aceitação da hipótese formulada. Provavelmente, o conteúdo informacional vinculado às alterações nos níveis de dividendos já foi absorvido anteriormente e não impactam na tomada de decisões dos fundos para aquisição de ações com essa característica.

Quanto ao fator book-to-market (BOOK), observa-se que o coeficiente $\beta$ apresentou significância para empresas com menor valor de mercado em relação ao valor contábil, em que os fundos preferem as empresas com maior diferença entre valor contábil e valor de mercado. É possível observar que o tamanho (VM) das empresas investidas de grande porte contribui positivamente pela maior procura dos fundos, seguido pelo critério de rentabilidade (RENT), que apresentou significância estatística com coeficiente $\beta$ positivo.

Diante dos resultados apresentados na Tabela 3, é possível inferir que os fundos de investimentos em ações apresentam estratégias para alocação de recursos nos moldes dos fatores delineados por Fama e French (2015) e Carhart (1997), com destaque à atratividade do 
fator dividendo. Esses resultados apontam para a não rejeição da hipótese H1a, indicando que $o$ atributo dividendo parece ser relevante para que os gestores realizem a alocação de recursos dos fundos que administram. As evidências encontradas corroboram com a teoria da sinalização dos dividendos (BENARTZI et al., 1997; DEANGELO; DEANGELO, 1990; GRULLON et al., 2002; MYERS; MAJLUF, 1984b; VAN HORNE; WACHOWICZ, 2005). Por outro lado, os resultados apontam para a não aceitação de H1b.

Em decorrência do resultado apresentado para a confirmação de atratividade dos fundos à distribuição de dividendos pelas empresas investidas, seguiu-se uma investigação com intuito de identificar a relação entre a distribuição de dividendos e o desempenho dos fundos (H2a). $\mathrm{O}$ Apêndice A apresenta os resultados para a regressão relacionando a performance dos fundos mútuos como variável dependente. O modelo 1, cujo VIF apresentou valor máximo de 19,94 entre as variáveis LIQ e VM, indicou que ambas estão altamente correlacionadas. Com isso, excluiu-se a variável LIQ gerando o modelo 2 que continuou apresentando VIF máximo de 5,62 na variável VM. Com o intuito de garantir a ausência de multicolinearidade, excluiu-se também a variável VM, conforme apresentado no modelo 3, cujo VIF máximo ficou em 2,6. Em complemento, foi estimado o modelo 4, considerando apenas as variáveis estatisticamente significativas para explicar o Índice de Sharpe dos fundos de investimentos. Este modelo apresentou VIF máximo de 2,1. Por fim, estimou-se o modelo 5 (VIF máximo em 2,95), em que a variável DIV foi substituída pela variável DIVADIC.

Análise para a hipótese $\mathrm{H} 2 \mathrm{a}$ está vinculada aos resultados apresentados no referido Apêndice A. Sobre o fator dividendo (DIV), não é possível afirmar sua relevância para a performance dos fundos, o que implica em não aceitação da hipótese H2a do presente estudo. Isso significa que o mercado pode já estar valorizando o conteúdo informativo dos dividendos em suas decisões estratégicas, e talvez por isso não seja possível detectar linearmente sua contribuição no retorno. Fundos parecem buscar ativos com distribuição de dividendos pelo prenúncio de bons resultados corporativos (conforme resultados observados para H1) independentemente de seu impacto no desempenho da carteira. Desta forma, o poder informativo do dividendo atrai os fundos, mas os ganhos extraordinários proporcionados pela negociação com base neste critério de decisão não parecem ser estatisticamente significativos, possivelmente em razão de diversos investidores institucionais estarem operando com base na mesma informação.

Por outro lado, a variável dividendo adicional (DIVADIC) apresentou efeito negativo. Esse achado indica que não necessariamente a rentabilidade disponibilizada pela empresa aos investidores por meio de dividendo é o que vai trazer melhor desempenho para o fundo, pois o preço da ação pode já ter absorvido aquela informação. Há uma reação negativa na performance dos fundos associada às mudanças positivas no dividendo. Uma potencial justificativa poderia ser de que esse incremento nos proventos aos acionistas interfere na expectativa de menor fluxo de caixa disponível para novos investimentos, conforme discutido pela literatura em relação ao tradeoff dividendo e investimento dividendos (GONZÁLEZ, 1998; GRAHAM; DODD, 1988; HAQUE; SIDDIKEE; HOSSAIN; CHOWDHURY; RAHMAN, 2013).

$\mathrm{O}$ fator book-to-market (BOOK) apresentou significância para o coeficiente $\beta$ referente às empresas com maior valor contábil em relação ao valor de mercado. Apesar das empresas se direcionarem pelo índice $\mathrm{B} / \mathrm{M}$, não necessariamente é um fator que acarreta um retorno ajustado ao risco positivo. Serra e Saito (2015) concluem que empresas com boas perspectivas sinalizam valor de mercado acima do valor patrimonial, refletindo elevado índice B/M. Novy-Marx (2013) afirma que empresas em que os investidores exigem altas taxas de retorno (ou seja, empresas de risco) estão com preços mais baixos e, consequentemente, apresentam maior índice $\mathrm{B} / \mathrm{M}$ do que empresas para as quais os investidores exigem retornos menores.

Comparando os resultados da Tabela 03 e do Apêndice A, há indícios de que os gestores de fundos de investimentos preferem alocar seus recursos em empresas com maior diferença 
entre valor contábil e valor de mercado (Tabela 03). Esta decisão parece também ser a que se associa com melhores indicadores de performance, pois fundos que investem naquelas ações com menor diferença entre valor contábil e valor de mercado apresentaram menor performance ajustada ao risco (Apêndice A).

O fator momentum representado pela variável RETP demonstrou significância para a performance dos fundos. As empresas com maior retorno médio contribuem positivamente para o desempenho dos investidores institucionais, ou seja, supostamente, o preço das ações esteja subestimado por novas informações publicadas, viabilizando a utilização de estratégias rentáveis pelos fundos baseadas na seleção de ações pelo seu retorno passado.

No fator investimentos (INV), houve significância com coeficiente positivo, indicando que elevado nível de investimentos das empresas investidas contribui favoravelmente para o incremento da performance dos fundos mútuos. Cabe destacar o contraponto entre investimentos e dividendos adicional em consonância com a literatura apresentada, demonstrando que o paradoxo é identificado nos resultados.

Em relação ao tamanho dos fundos, Milani e Ceretta (2013) confirmam, assim como o presente estudo, o efeito tamanho no Brasil apontando que fundos maiores tendem a apresentar melhor performance, assim como apontado por Indro et al (1999). Quanto à idade (AGE), os fundos com maior tempo de atuação no mercado contribuíram negativamente para maior performance dos fundos contrariando Milani e Ceretta (2013) no contexto brasileiro e em consonância à literatura internacional (GRINBLATT; TITMAN, 1989).

\section{CONSIDERAÇÕES FINAIS}

O objetivo proposto foi investigar a relação do pagamento de dividendos com a composição de carteiras e a performance dos fundos no Brasil. Para tanto, o modelo de cinco fatores sintetizados por Fama e French $(1993,2015)$ e Carhart (1997) foi adaptado.

Embora o conteúdo informacional dos dividendos ainda seja um puzzle, os resultados encontrados, inicialmente, apontam para a não rejeição de H1a, em que o fator dividendo foi significativo a $5 \%$, ou seja, os fundos de investimentos em ações (em média) utilizam a informação proveniente de dividendos para decidir sobre composição de suas carteiras. Essa constatação, possivelmente, é proveniente da sinalização emitida ao mercado da credibilidade e qualidade organizacional com o pagamento de dividendos, consequentemente, menor risco na alocação de recursos, conforme apontado pela revisão da literatura. Portanto, apesar de não ser considerado em modelos consolidados na literatura, o fator dividendo é importante para a tomada de decisões de gestores dos fundos na formação do portfólio. Entretanto, os resultados apontam para a não aceitação de $\mathrm{H} 1 \mathrm{~b}$, indicando que as ações com variações nos níveis de dividendos não impactam na atratividade dos fundos.

Cabe salientar que as características que mais se destacaram, para a composição das carteiras, apresentando os maiores coeficientes $\beta$ foram BOOK (book-to-market) e VM (tamanho), seguido por RENT (rentabilidade), enquanto as variáveis RENT (rentabilidade) e DIV (dividendo) se apresentaram como bons direcionadores É uma indicação de que os investidores institucionais, mesmo em mercados emergentes, buscam estratégias com foco nesses fatores. O presente estudo de fundos mútuos fornece importantes insights sobre as percepções generalizadas de que os investidores institucionais possuem informação superior, que por sua vez leva à preferência ou não de empresas em sua carteira. Essa informação pode permitir aos investidores institucionais estruturar suas carteiras de acordo com sua preferência de risco e retorno.

Ao analisar a relação entre o pagamento de dividendos das empresas investidas e o desempenho dos fundos (H2a), observou-se que esse fator não apresentou significância estatística. Os fundos utilizam a informação sobre os dividendos para a composição de suas carteiras e essa informação parece ser compreendida, apesar de não ser possível afirmar sobre 
o impacto em sua performance. Esse impacto não significativo na performance pode estar vinculado à exploração simultânea da mesma informação entre vários investidores institucionais.

Os resultados têm implicações para a comunidade acadêmica e profissionais de mercado que procuram aumentar seus retornos sobre os investimentos, pois evidenciou-se uma variável que pode ser relevante para testes empíricos de teorias e para alocação de recursos por investidores institucionais. Complementa-se a literatura de economias emergentes ao evidenciar que fundos de investimentos em ações podem se orientar pelo efeito clientela de dividendos. Confirmou-se o efeito clientela em dividendos por parte dos fundos mútuos de ações no Brasil, assim como Holanda e Coelho (2012), bem como a contribuição de seu incremento na performance desses investidores institucionais. Enfim, evidenciou-se uma variável capaz de capturar o retorno anormal de fundos mútuos no Brasil, sendo de relevância prática para a gestão e a precificação de ativos, bem como ao gerenciamento de risco.

Os resultados podem ajudar gestores e investidores a construir suas previsões de retorno e selecionar os modelos de avaliação com maior precisão. A amplitude do presente estudo com a evidenciação de mais uma dimensão importante às estratégias dos fundos fortalece a necessidade de novas investigações para consolidar todas as anomalias atreladas ao norteamento dos fundos brasileiros na alocação de recursos em ativos mobiliários.

Faz-se necessário comentar uma limitação do presente estudo, uma vez que a literatura de suporte utilizada para a elaboração das hipóteses é majoritariamente proveniente de estudos sobre finanças corporativas, e não necessariamente relacionando dividendos a fundos mútuos. Embora exista essa limitação, foi possível detectar uma relação positiva e significativa entre dividendos e composição de carteiras dos fundos, e indicar que não necessariamente a informação sobre dividendos se converte em melhor performance ajustada ao risco, abrindo, desta forma, oportunidade para novas pesquisas sobre o assunto.

\section{AGRADECIMENTOS}

Rodrigo F. Malaquias agradece à FAPEMIG pelo apoio concedido para a realização de parte desta pesquisa (Projeto de Demanda Universal, 2014, APQ-01265-14).

\section{REFERÊNCIAS}

AJINA, A.; LAKHAL, F.; SOUGNÉ, D. Institutional investors, information asymmetry and stock market liquidity in France. International Journal of Managerial Finance, vol. 11, n. 1, p. 44-59. 2015. http://doi.org/10.1108/IJMF-08-2013-0086

BAKER, H. K. Dividends and dividend policy. (Kolbseries). New Jersey: JohnWiley \& Sons. 2009. http://doi.org/10.1007/s13398-014-0173-7.2

BAKER, M.; NAGEL, S.; WURGLER, J. The Effect of Dividends on Consumption. Brookings Papers on Economic Activity, 2007(1), p. 231-291. 2007. http://doi.org/10.1353/eca.2007.0010

BAKER, M.; WURGLER, J. A Catering Theory of Dividends. The Journal of Finance, vol. 59, n. 3, p. 1125-1165. 2004.

BENARTZI, S.; MICHAELY, R.; THALER, R. Do changes in dividends signal the future or the past? The Journal of Finance, vol. 52, n. 3, p. 1007-1034. 1997.

http://doi.org/10.2307/2329514 
DIVIDENDOS, COMPOSIÇÃO DE CARTEIRAS E PERFORMANCE DE FUNDOS DE AÇÕES

BHATTACHARYA, S. Imperfect Information, Dividend Policy, and "The Bird in the Hand" Fallacy. The Bell Journal of Economics, vol. 10, n. 1, p. 259-270. 1979. http://doi.org/10.2307/3003330

BLACK, F.; SCHOLES, M. The effects of dividend yield and dividend policy on common stock prices and returns. Journal of Financial Economics, vol. 1, n. 1, p. 1-22. 1974. http://doi.org/10.1016/0304-405X(74)90006-3

BORGES, E. C.; MARTELANC, R. Sorte ou habilidade: uma avaliação dos fundos de investimento no Brasil. Revista de Administração, vol. 50, n. 2, p. 196-207. 2015. http://doi.org/10.5700/rausp1194

BREALEY, R. A.; MYERS, S. C.; ALLEN, F. Principles of Corporate Finance (10th ed.). New York: McGraw-Hill. 2011.

CALVET, L. E.; FISHER, A. J. Multifractual volatility: theory, forecasting and pricing. Monographs of the Society for Research in Child Development (Vol. 78). Amsterdam: Academic Press. 2008. http://doi.org/10.1111/mono.12025

CARHART, M. M. On Persistence in Mutual Fund Performance. The Journal of Finance, vol. 52, n. 1, p. 57-82. 1997.

http://doi.org/http://www.blackwellpublishing.com/journal.asp?ref=0022-1082

CASTRO, B. R.; MINARDI, A. M. A. F. Comparação do Desempenho dos Fundos de Ações Ativos e Passivos. Revista Brasileira de Finanças, vol. 7, n. 2, p. 143-161. 2009.

DANIEL, K.; GRINBLATT, M.; TITMAN, S.; WERMERS, R. Measuring mutual fund performance with characteristic based benchmarks. The Journal of Finance, vol. 52, n. 3, p. 1035-1058. 1997. http://doi.org/10.1111/j.1540-6261.1997.tb02724.x

DEANGELO, H.; DEANGELO, L. Dividend Policy and Financial Distress: An Empirical Investigation of Troubled NYSE Firms. The Journal of Finance, vol. 45, n. 5, p. 1415-1431. 1990. http://doi.org/10.1111/j.1540-6261.1990.tb03721.x

DEANGELO, H.; DEANGELO, L.; SKINNER, D. J. Dividends and Losses. The Journal of Finance, vol. 47, n. 5, p. 1837-1863. 1992. http://doi.org/10.1111/j.15406261.1992.tb04685.x

DENG, J.; CHAN, D. K.; YANG, S. X.; CAUDWELL, K. M. Proceedings of the Ninth International Conference on Management Science and Engineering Management. (J. Xu, S. Nickel, V. C. Machado, \& A. Hajiyev, Eds.) (Vol. 362). Berlin, Heidelberg: Springer Berlin Heidelberg. 2015. http://doi.org/10.1007/978-3-662-47241-5

DENIS, D. J.; DENIS, D. K.; SARIN, A. The Information Content of Dividend Changes: Cash Flow Signaling, Overinvestment, and Dividend Clienteles. Journal of Financial and Quantitative Analysis, vol. 29, n. 4, p. 567-587. 1994. http://doi.org/10.2307/2331110

DÍAZ-MENDOZA, A. C.; LÓPEZ-ESPINOSA, G.; MARTÍNEZ, M. A. The Efficiency of Performance-Based Fee Funds. European Financial Management, vol. 20, n. 4, p. 825-855. 2014. http://doi.org/10.1111/j.1468-036X.2012.00654.x 
EVANS, A. L. Portfolio Manager Ownership and Mutual Fund performance. Financial Management, vol. 37, n. 3, p. 513-534. 2008.

FAMA, E. F.; FRENCH, K. R. Dividend yields and expected stock returns. Journal of Financial Economics, vol. 22, n. 1, p. 3-25. 1988. http://doi.org/10.1016/0304405X(88)90020-7

FAMA, E. F.; FRENCH, K. R. Common risk factors in the returns on stocks and bonds. Journal of Financial Economics, vol. 33, n. 1, p. 3-56. 1993. http://doi.org/10.1016/0304405X(93)90023-5

FAMA, E. F.; FRENCH, K. R. A five-factor asset pricing model. Journal of Financial Economics, vol. 116, n. 1, p. 1-22. 2015. http://doi.org/10.1016/j.jfineco.2014.10.010

FÁVERO, L. P. Análise de Dados (1st ed.). Rio de Janeiro: Elsevier. 2015.

FORTI, C. A. B.; PEIXOTO, F. M.; ALVES, D. L. Determinant Factors of Dividend Payments in Brazil. Revista Contabilidade \& Finanças, vol. 26, n. 68, p. 167-180. 2015. http://doi.org/10.1590/1808-057x201512260

GIL-BAZO, J.; RUIZ-VERDÚ, P. The Relation between Price and Performance in the Mutual Fund Industry. The Journal of Finance, vol. 64, n. 5, p. 2153-2183. 2009. http://doi.org/10.1111/j.1540-6261.2009.01497.x

GONZÁlEZ, P. G. As Mudanças nas Políticas de Dividendos e o Mercado Financeiro. Caderno de Estudos, vol. 10, n. 19, p. 70-81. 1998.

GRAHAM, B.; DODD, D. Security analysis (6th ed.). New York: Mc Graw Hill. 1988.

GRINBLATT, M.; TITMAN, S. Mutual Fund Performance: An Analysis of Quarterly Portfolio Holdings. The Journal of Business, vol. 62, n. 3. 1989.

http://doi.org/10.1086/296468

GRULLON, G.; MICHAELY, R.; SWAMINATHAN, B. Are Dividend Changes a Sign of Firm Maturity? The Journal of Business, vol. 75, n. 3, p. 387-424. 2002.

http://doi.org/10.1086/339889

GUARANA, L. Estudo do impacto do patrimônio na rentabilidade dos fundos de investimentos em ações. Dissertação (EPGE - Escola de Pós-Graduação em Economia), Fundação Getúlio Vargas. 2012.

GUERCIO, D. D.; TKAC, P. A. The Determinants of the Flow of Funds of Managed Portfolios: Mutual Funds vs. Pension Funds. The Journal of Financial and Quantitative Analysis, vol. 37, n. 4, p. 523-557. 2002. http://doi.org/10.2307/3595011

HAQUE, R.; SIDDIKEE, J. A.; HOSSAIN, S.; CHOWDHURY, S. P.; RAHMAN, M. Relationship between dividend payout and Economic Value Added: A case of Square Pharmaceuticals Limited, Bangladesh. International Journal of Innovation and Applied Studies, vol. 3, n. 1, p. 98-104. 2013. 
HOLANDA, A. P.; COELHO, A. C. D. Dividendos e efeito clientela: evidências no mercado brasileiro. Revista de Administração de Empresas, vol. 52, n. 4, p. 448-463. 2012. http://doi.org/10.1590/S0034-75902012000400007

INDRO, D. C.; JIANG, C. X.; HU, M. Y.; LEE, W. Y. Mutual Fund performance: does fund size matter? Financial Analysts Journal, vol. 55, n. 3, p. 74-87. 1999.

ISRAELSEN, C. L. A refinement to the Sharpe ratio and information ratio. Journal of Asset Management. v. 5, 6. 423-427. 2005.

JABBOURI, I. Determinants of corporate dividend policy in emerging markets: Evidence from MENA stock markets. Research in International Business and Finance, vol. 37, p. 283-298. 2016. http://doi.org/10.1016/j.ribaf.2016.01.018

JAIN, P. C.; WU, J. S. Truth in Mutual Fund Advertising: Evidence on Future Performance and Fund Flows. The Journal of Finance, vol. 55, n. 2, p. 937-958. 2000.

http://doi.org/10.1111/0022-1082.00232

JENSEN, M. C. The Performance of Mutual Funds in the Period 1945-1964. The Journal of Finance, v. 23, n. 2, 389-416, 1968. doi: https://doi.org/10.1111/j.1540-6261.1968.tb00815.x

LINTNER, J. Distribution of incomes of corporations among dividends, retained earnings, and taxes. American Economic Review, v. 46, n. 2, p. 97-113. 1956.

MARKOWITZ, H. M. Portfolio Selection. Journal of Finance, vol. 7, n. 1, 1952.

MILANI, B.; CERETTA, P. S. Efeito tamanho nos fundos de investimento brasileiros.

Revista de Administração Da UFSM, vol. 6, n. 1, p. 119-138. 2013.

http://doi.org/10.5902/198346593607

MOREIRAS, L. M. F.; TAMBOSI FILHO, E.; GARCIA, F. G. Dividendos e informação assimétrica: análise do novo mercado. Revista de Administração, vol. 47, n. 4, p. 671-682. 2012. http://doi.org/10.5700/rausp1066

MYERS, S. C.; MAJLUF, N. S. Corporate financing and investment decisions when firms have information that investors do not have. Journal of Financial Economics, vol. 13, n. 2, p. 187-221. 1984a. http://doi.org/10.1016/0304-405X(84)90023-0

MYERS, S. C.; MAJLUF, N. S. Corporate financing and investment decisions when firms have information that investors do not have. Journal of Financial Economics, vol. 13, n. 2, p. 187-221. 1984b. http://doi.org/10.1016/0304-405X(84)90023-0

NIKOLAOS, E.; EVANGELOS, P. I.; DIMITRIOS, V. The dividend policy of the Greek listed firms prior the economic crisis: A comparative analysis. Paper Presented at the 9 Annual Conference, European Economics and Finance Society, Athens, Greece. 2010.

NOVY-MARX, R. The other side of value: The gross profitability premium. Journal of Financial Economics, vol. 108, n. 1, p. 1-28. 2013.

http://doi.org/10.1016/j.jfineco.2013.01.003 
PROCIANOY, J. L.; VERDI, R. S. Dividend Clientele, new insights, and new questions: the Brazilian case. Revista de Administração de Empresas - Eletrônica, vol. 8, n. 1, Art. 1. 2009. http://doi.org/10.1017/CBO9781107415324.004

RAMALINGEGOWDA, S.; WANG, C. S.; YU, Y. The role of financial reporting quality in mitigating the constraining effect of dividend policy on investment decisions. Accounting Review, vol. 88, n. 3, p. 1007-1039. 2013. http://doi.org/10.2308/accr-50387

REILLY, F. K.; BROWN, K. C. Investment Analysis \& Portfolio Management (10th ed.). South-Western: Cengage Learning. 2012.

SANTOS. J. O.; SILVA, R. O. R. C.; COELHO, P. A. R.; GIACCHERI, D. Conteúdo informacional: uma análise da relação entre lucros e dividendos. In: XI Seminários em Administração. 2008, São Paulo. Anais... FEA/USP. São Paulo: USP, 2008.

SCHOLZ, J. K. A direct examination of the dividend clientele hypothesis. Journal of Public Economics, vol. 49, n. 3, p. 261-285. 1992. http://doi.org/10.1016/0047-2727(92)90069-R

SERRA, R. G.; SAITO, A. T. Determinantes do P/B, setor regulado e estratégias de investimento. Revista de Administração FACES Journal, vol. 15, n. 1, p. 82-96. 2015. http://doi.org/10.1017/CBO9781107415324.004

SHARPE, W. F. Mutual Fund Performance. The Journal of Business, vol. 39, n. 1, p. 119138. 1966. Retrieved from http://www.jstor.org/stable/2351741

SILVA, A. L. C. Governança corporativa, valor, alavancagem e política de dividendos nas empresas brasileiras. Revista de Administração, vol. 39, n. 4, p. 348-361. 2004.

VAN HORNE, J. C.; WACHOWICZ, J. M. Fundamentals of Financial Management (12th ed.). New Jersey: Financial Times Prentice Hall. 2005. Retrieved from https://books.google.com.br/books?id=CPGC4pD2pQ0C

VIEIRA, E.; PINHO, C.; LEITE, S. Reação do Mercado ao Anúncio de Dividendos:

Evidência em Países Europeus. Estudos Do ISCA, vol. 4, n. 5, p. 1-14. 2013.

WATTS, R. (1973). The Information Content of Dividends. The Journal of Business, vol. 46, n. 2, p. 191-211. 1973. http://doi.org/10.1086/295525 
DIVIDENDOS, COMPOSIÇÃO DE CARTEIRAS E PERFORMANCE DE FUNDOS DE AÇÕES

Apêndice A. Análise da relação entre dividendos e performance dos fundos da amostra

\begin{tabular}{|c|c|c|c|c|c|c|c|c|c|c|c|c|c|c|c|}
\hline \multirow{2}{*}{$\begin{array}{l}\text { Painel: } \\
\text { Variáveis }\end{array}$} & \multicolumn{3}{|c|}{01} & \multicolumn{3}{|c|}{02} & \multicolumn{3}{|c|}{$\mathbf{0 3}$} & \multicolumn{3}{|c|}{04} & \multicolumn{3}{|c|}{05} \\
\hline & Coef. & $\mathbf{t}$ & $P>t$ & Coef. & $\mathbf{t}$ & $\mathbf{P}>\mathbf{t}$ & Coef. & $\mathbf{t}$ & $\mathbf{P}>\mathbf{t}$ & Coef. & $\mathbf{t}$ & $P>t$ & Coef. & $\mathbf{t}$ & $\mathbf{P}>\mathbf{t}$ \\
\hline LIQ & -0.057 & -3.73 & 0.000 & --- & --- & --- & --- & --- & --- & --- & --- & --- & --- & --- & --- \\
\hline VM & 0.042 & 2.78 & 0.005 & -0.006 & -0.74 & 0.457 & --- & --- & --- & --- & --- & --- & --- & --- & --- \\
\hline BOOK & -0.097 & -17.86 & 0.000 & -0.102 & -19.44 & 0.000 & -0.103 & -21.97 & 0.000 & -0.105 & -22.38 & 0.000 & -0.080 & -16.32 & 0.000 \\
\hline INV & 0.083 & 15.63 & 0.000 & 0.081 & 15.32 & 0.000 & 0.080 & 16.07 & 0.000 & 0.078 & 16.17 & 0.000 & 0.041 & 7.73 & 0.000 \\
\hline RENT & -0.024 & -3.89 & 0.000 & -0.025 & -4.17 & 0.000 & -0.027 & -4.46 & 0.000 & -0.030 & -5.32 & 0.000 & 0.029 & 4.32 & 0.000 \\
\hline RETP & 0.035 & 4.77 & 0.000 & 0.034 & 4.60 & 0.000 & 0.034 & 4.60 & 0.000 & 0.031 & 4.36 & 0.000 & 0.055 & 7.74 & 0.000 \\
\hline BETA & 0.058 & 11.77 & 0.000 & 0.056 & 11.36 & 0.000 & 0.055 & 11.82 & 0.000 & 0.055 & 11.82 & 0.000 & 0.066 & 14.46 & 0.000 \\
\hline DIV & -0.005 & -0.86 & 0.389 & -0.008 & -1.22 & 0.223 & -0.009 & -1.56 & 0.118 & --- & --- & --- & --- & --- & --- \\
\hline DIVADIC & --- & --- & --- & --- & --- & --- & --- & --- & --- & --- & --- & --- & -0.098 & -14.61 & 0.000 \\
\hline LNPL & 2.171 & 14.50 & 0.000 & 2.175 & 14.51 & 0.000 & 2.177 & 14.53 & 0.000 & 2.186 & 14.84 & 0.000 & 2.161 & 14.98 & 0.000 \\
\hline AGE & -1.729 & -35.38 & 0.000 & -1.717 & -35.16 & 0.000 & -1.712 & -35.33 & 0.000 & -1.695 & -35.73 & 0.000 & -1.668 & -35.87 & 0.000 \\
\hline LOCKUP & 0.058 & 3.11 & 0.002 & 0.057 & 3.08 & 0.002 & 0.057 & 3.08 & 0.002 & 0.068 & 4.02 & 0.000 & 0.062 & 3.73 & 0.000 \\
\hline TXADM & 1.175 & 1.80 & 0.073 & 1.190 & 1.82 & 0.070 & 1.204 & 1.84 & 0.066 & 1.788 & 3.39 & 0.001 & 1.728 & 3.35 & 0.001 \\
\hline TXPERF & 2.237 & 1.21 & 0.227 & 2.327 & 1.26 & 0.209 & 2.390 & 1.29 & 0.197 & --- & --- & --- & --- & --- & --- \\
\hline _cons & -35.657 & -14.23 & 0.000 & -35.963 & -14.34 & 0.000 & -36.134 & -14.47 & 0.000 & -36.546 & -14.78 & 0.000 & -35.905 & -14.83 & 0.000 \\
\hline
\end{tabular}

Notas: Variável dependente (SHARPE) relacionada com as variáveis independentes: liquidez em bolsa (LIQ), valor de mercado (VM), book-to-market (BOOK), investimentos (INV), rentabilidade (RENT), retorno passado (RETP), risco sistemático (BETA), dividendos (DIV) e dividendo adicional (DIVADIC), tamanho (LNPL), idade (AGE), taxa de performance (TXPERF), taxa de administração (TXADM), período de lock-up (LOCKUP). 